UDC 378

DOI: https://doi.org/10.24195/2414-4665-2019-4-1

Estella W. Chizhik,

Professor of Teacher Education, San Diego State University, San Diego, USA

Alexander W. Chizhik,

Professor of Teacher Education, San Diego State University, San Diego, USA

Brian Burgess,

Student in the Psychology Department, San Diego State University, San Diego, USA

Maelyn Tanmajo,

B.A. in Psychology, San Diego State University, San Diego, USA

Derrick Seng,

Student in the Psychology Department, San Diego State University, San Diego, USA

Myrna Hernandez,

B.A. in Psychology, San Diego State University, San Diego, USA

*Corresponding authoremail: chizhik@sdsu.edu

\title{
VIDEO-BASED COACHING IN SUPPORT OF ELEMENTARY TEACHER-CANDIDATE'S PROFESSIONAL DEVELOPMENT
}

Teacher-education programs seek to improve their clinical practice for teacher candidates. Video-based coaching enables university supervisors the ability to provide meaningful feedback to teacher candidates. Most of the research on video-based coaching has focused on how the tool helps candidates notice aspects of their teaching. Few studies have examined the type of feedback university supervisors provide their teacher candidates. The current research examined the type of feedback university supervisors provide, how the feedback changes over the course of the program and its impact on a summative performance-based assessment. Reviewing the feedback provided by 16 university supervisors for 124 elementary school teacher candidates, our findings show that university supervisors' feedback tends to be more positive than constructive. The select skills on which supervisors focused modulated over time and appear to be associated with candidates' performance on the summative performance assessment. The implications of this research posits that university supervisors can have a measurable effect on teacher candidates' instructional performance with the use of video-based coaching.

Keywords: teacher education, teacher candidates, university supervisors, video-based coaching, feedback, performance-based assessment

\section{Introduction \& Purpose}

Clinical practice plays a critical role in preparing future educators to teach in diverse classrooms (Koerner, Rust, \& Baumgartner, 2002). Perhaps because of this, the California Commission on Teacher Credentialing (CCTC) updated its standards on field experiences and placed greater emphasis on supervision of teacher-candidates. This study, therefore, focuses on a teacher education program in California, USA, that transformed the supervision model to conform to the updated CCTC standards by including a video-based coaching tool, known as Sibme. This video-based coaching tool leverages supervisors' ability to provide more relevant and useful feedback to teacher-candidates. Feedback includes detailed commentary and "tags" to specify "essential pedagogical skills" to guide supervisors' feedback (see Table 1). These skills are based on CCTC's Teacher-Performance Expectations and are connected to edTPA Instructional Rubrics, a highstakes performance assessment for teacher-candidates that is widely used throughout the United States. With this Sibme-based structured feedback, teacher-candidates can review their teaching and supervisors' comments at critical moments when incidents occur on video. For example, if university-supervisors notice teacher-candidates' utilizing particularly effective scaffolds at minute six and 43 seconds of the video, supervisors can select corresponding pedagogical skills, and write comments that point to that specific location on the video.
Prior to program-wide adoption of Sibme, two of the researchers conducted a pilot study with a cohort (30 teacher-candidates). We asked teacher-candidates to evaluate effectiveness of Sibme feedback relative to traditional forms of feedback. Candidates reported that Sibme-based feedback from supervisors helped them see strengths and weaknesses that were difficult to identify during face-toface feedback (Authors, 2017; Authors, 2018). While the study addressed teacher-candidates' perceptions of the video-based coaching tool, it did not examine the quality of the feedback and how that feedback affected instructional performance. As a result, the current research study examines the quality of feedback supervisors offer to elementary teacher-candidates; whether this feedback changes over the course of the teacher education program, and whether there is a relationship between the feedback and teacher-candidates' instructional performance. Specifically, we had the following questions:

1) What "essential pedagogical skills" are universitysupervisors targeting when providing feedback to elementary teacher-candidates' through Sibme? 2) To what extent do "essential pedagogical skills" targeted by university-supervisors' change as teacher-candidates' gain experience over the course of an academic year?

3) What relationship is there between university-supervisors' feedback and teacher-candidates' performance on the edTPA, a summative performance assessment that is widely used throughout the United States? 


\section{Relevant Literature \& Theoretical Framework}

Being able to pause and repeatedly watch video-recorded teaching affords teacher-candidates' opportunities for a focused analysis of their teaching (Tan \& Towndrow, 2009). With video, teacher-candidates' can be guided by experts (e.g., mentor teachers, university-supervisors, school administrators) to notice, reflect, and reconsider specific moments and aspects of their practice (Hamilton, 2012; van Es \& Sherin, 2008). Opportunities to deconstruct instructional lessons have potential benefits of gaining insight into the challenges of teaching, prompting problem-solving, and producing changes in practice (Marsh \& Mitchell, 2014). Research focusing on videobased coaching has typically examined its effects on teacher performance (Sun \& van Es, 2015). Our research aims to focus specifically on examination of the feedback offered through video-based coaching tools and is based on a combination of two theoretical frameworks. The first framework draws upon research on feedback, defined as, "information provided by teachers concerning performance or understanding of students, with reference to a goal and aimed at improving learning" (Voerman et al., 2012). Effective feedback can be positive and/or critical as long as it is specific (Hattie \& Timperley, 2007; Shute, 2008; Voerman et al., 2012). The level of specificity is framed by our second theoretical framework, professional vision (Goodwin, 1994) and noticing (Mason, 2002). According to Goodwin (Goodwin, 1994), professional vision focuses on the knowledge and skills associated with a profession. Therefore, the professional vision can highlight skills and knowledge associated with the practice of teaching (Lefstein and Snell, 2011) and student learning outcomes (Stürmer, Knönings, \& Seidel, 2013). Feedback, therefore, should be designed to develop this professional vision. Mason's (2002) work on "noticing" illuminates how professional visions develop in teaching. Supporting teachers noticing relies on experts in the field helping them "notice" the essential skills and knowledge associated with the profession. In education, support providers, like university-supervisors', enable candidates to notice specific instructional behaviors, statements, or movements in the classroom.

These similar constructs suggest that experts mediate novices' development by "highlighting" key concepts or by helping candidates "notice" undetected aspects of their teaching or classroom environments. These theoretical frameworks afford us the lens to examine pedagogical skills that supervisors target in providing feedback to help teacher-candidates notice instructional activities and behaviors that support construction of their own teaching knowledge (Wu \& Lee, 2004). Specificity of feedback in student teaching, therefore, can play a role in developing teacher-candidates' pedagogy. Defining that specificity of feedback and examining its relationship to teacher-candidates' instructional performance frames the goals of this research.

\section{Research Methods. Data Sources Overview}

Data for this study consisted of teacher-candidates' video-based recordings of student-teaching in public-edu- cation classes, university-supervisors' written feedback associated with these recordings, and teacher-candidates' scores on the national teacher-performance assessment (edTPA). Data was collected with teacher-candidates' and university-supervisors' informed consent. Teacher-candidates' consent was acquired after their completion of the two-semester teacher education program. One-hundredtwenty-four elementary teacher-candidates and their 16 university-supervisors participated in this research.

Video-based Recordings

Teacher-candidates recorded approximately for five to six student-teaching lessons during the year-long teacher-credential program. The duration of each video was between 15 to 45 minutes. University-supervisors observed their teacher-candidates' videos and provided feedback using Sibme, a video-based coaching tool. University-supervisors could provide several types of feedback. The first type of feedback focused on giving three general feedback tags: bright spots, ideas, and questions. These "tags" informed candidates regarding instructional behaviors at which they excel (e.g., bright spots), recommendations to consider for future lessons (e.g., ideas), and behaviors or thoughts about which teacher-candidates should think critically (e.g., questions). For example, when a university-supervisor saw a behavior that is good and should be repeated in future lessons, the supervisor used a "bright spot" tag. When a university-supervisor saw a teaching behavior about which the teacher candidate should think critically, the university-supervisor used the "question" tag and wrote the question in the space provided. The written feedback was marked at the point in the video where the concern occurred.

In addition to the three general feedback tags, university-supervisors could give more specific feedback tags that addressed 30 pedagogical skills. One of the authors of this paper and a university-supervisor identified a list of "essential pedagogical skills" that are aligned with the California standards for teacher education and support candidates' performance on the edTPA, a performance assessment that is widely used throughout the United States. These 30 tags are designed to highlight specific behaviors that university-supervisors can help their teacher-candidates' notice. The pedagogical skills focus on teachers' construction of the learning environment (e.g., rapport and the candidate's strong voice), methods to engage students (e.g., maintaining students' attention, modeling, and positive feedback), deepening students' thinking (e.g., building on students responses, student self-reflection, asking questions), and specific pedagogical practices (e.g., technology, academic language, and pacing). These skills (see Table 1) were accessible on Sibme for university-supervisors to use as "tags." When university-supervisors saw a pedagogical skill being used or thought that one should be used, they could use the appropriate tag and provide detailed feedback at the designated time in the video. Using these 30 tags that highlight pedagogical skills, we examined how specific feedback (the tags) support teacher-candidates' professional development. 
Link between edTPA Instructional Rubrics and Sibme Essential Pedagogical Skills

edTPA Instructional Rubrics

Sibme Essential Pedagogical Skills

\begin{tabular}{ll}
\hline Learning Environment & Rapport, Positive Language, Restorative practices, \\
& Behavior expectations, Redirection, Scans Room, Strong Voice
\end{tabular}

Engaging Students in Learning Maintains student attention, Modeling, Relevant Connections,

Asking Questions/Checks for Understanding, Wait time,

Student-to-Student Interactions, Peer-Evaluation, Technology, Many

Participate, Positive Feedback

Deepening Student Learning

Builds on Students' Responses, Student Self-Reflection,

Asking Questions/Checks for Understanding, Peer-Evaluation, Student-to-

Student Interactions, Practice Opportunities,

Challenging Learning Environment, Feedback to Many,

Timely Feedback, Teachable Moments

Subject Specific Pedagogy

Technology, Academic Language, Integrated and Designated English Language Development, Sequencing, Teachable Moments, Pacing, Instructional Activities

\section{Performance-based Assessment}

In California, all teacher-candidates' must successfully complete a teaching performance assessment, like the edTPA, in order to earn a teaching credential. The assessment consists of four tasks. Three tasks (Tasks 1-3) are associated with reading and language arts (Planning, Instruction, and Assessment). Task 4 is associated with mathematics. For Task 1, candidates submit three to five lesson plans and commentaries explaining their planned decisions regarding their instruction. For Task 2, candidates submit two videos of their instruction, totaling no more than 20 minutes. Task 3 requires candidates to describe their class's performance on an assessment along with three examples of students' work. For Task 4, candidates must submit descriptions of mathematics lessons, their students' performance on these lessons, and descriptions of re-engagement lessons based on students' performance on formative assessments. Teacher-candidates submit these components to Pearson (a private company) that hires and trains reviewers to score the portfolios. Candidates' submissions are scored on 18 rubrics (five rubrics for Tasks 13 and three rubrics for Task 4). Each rubric is scored on a five-point rating scale. Generally, scores of three and above demonstrate competency.

For this study, we were interested in the connection between university-supervisors' feedback during the academic year and teacher-candidates' performance on the edTPA. Thus, our analyses focused on portions of the edTPA where teacher-candidates demonstrated their instructional skills, specifically rubrics six through nine of Task 2. These rubrics focus on candidates' learning environment (rubric 6), engaging students in learning (rubric 7), deepening student learning (rubric 8), and literacy specific pedagogy (rubric 9). Our analyses focused on rubrics
6-9 because Pearson reviewers rated candidates on their teaching as observed in the two videos.

\section{Analyses and Results}

This research explored three questions addressing university-supervisors' feedback to teacher-candidates. First, we wanted to know which pedagogical skills did university-supervisors use to provide meaningful feedback. Specifically, we wanted to know whether there were pedagogical skills on which university-supervisors focused more frequently when using video-based coaching and whether there were pedagogical skills less frequently discussed during feedback. Second, we asked to what extent do "essential pedagogical skills" targeted by university-supervisors change as teacher-candidates gain experience over the course of an academic year? Finally, we asked, what effect does university-supervisors' feedback have on candidates' performance on the summative assessment known as the edTPA?

1) What "essential pedagogical skills" are university-supervisors targeting when providing feedback to elementary teacher-candidates through Sibme?

To determine if there were differences in the amount of each type of feedback that university-supervisors provided to teacher-candidates, we conducted two Repeated Measures ANOVAs and associated post-hoc comparisons. The first analysis used three within-subject levels that included the three general categories of feedback: bright spots, ideas, and questions. This analyses detected statistically significant differences, $F(2)=122.98, p<.001$. Posthoc analyses revealed statistically significant differences among all three types of feedback (see Table 2). University-supervisors used "bright spots" more than any other general tag. Supervisors used "ideas" more than "questions." 
Comparative Differences Among General Feedback Codes

\begin{tabular}{lcccc}
\hline \multicolumn{1}{c}{ Feedback $\mathrm{k}^{\mathrm{a}}$} & $M(S D)$ & Bright Spots & Ideas & Questions \\
& & & & \\
\hline Bright Spots & $30.20(16.93)$ & - & $* * *$ & \\
Ideas & $13.62(9.56)$ & $* * *$ & - & $* * *$ \\
Questions & $6.73(5.96)$ & $* * *$ & $* * *$ & - \\
\hline
\end{tabular}

$$
\begin{aligned}
& { }^{\mathrm{a}} n=79 . \\
& * * * p<.001 .
\end{aligned}
$$

The second analysis used 30 within-subject levels that included the 30 specific categories of feedback (see Table 3). These analyses detected statistically significant
Table 2 
$=2.30, p<0.01$. The associated paired t-tests are reported in Table 4.

Analyses revealed that university-supervisors used some "tags" routinely throughout the year (e.g., bright spots), some more in the fall than in the spring (e.g., ideas, movement around the room, scans room), and others more in the spring than in the fall (e.g., peer evaluation, relevant connection). It is important to note that not every teachercandidate received feedback on each of the skills. These findings show that university-supervisors modulate their feedback over time; increasing their focus on some skills and decreasing their focus on others.

Differences in Mean (SD) of Feedback Based on Semester

\begin{tabular}{|c|c|c|c|c|}
\hline Feedback $^{\mathrm{a}}$ & Semester & $M(S D)$ & $\begin{array}{l}\text { Paired } \\
\text { t-test }\end{array}$ & $p$ \\
\hline Bright Spots & $\begin{array}{l}\text { Fall } \\
\text { Spring }\end{array}$ & $\begin{array}{l}14.87(10.66) \\
15.33(11.37)\end{array}$ & 0.29 & $n s$ \\
\hline Ideas & $\begin{array}{l}\text { Fall } \\
\text { Spring }\end{array}$ & $\begin{array}{l}7.67(6.59) \\
5.95(4.72)\end{array}$ & 2.42 & $*$ \\
\hline Questions & $\begin{array}{l}\text { Fall } \\
\text { Spring }\end{array}$ & $\begin{array}{l}3.66(3.71) \\
3.08(4.02)\end{array}$ & 1.05 & $n s$ \\
\hline Academic Language & $\begin{array}{l}\text { Fall } \\
\text { Spring }\end{array}$ & $\begin{array}{l}1.61(2.53) \\
1.91(1.91)\end{array}$ & 0.43 & $n s$ \\
\hline Acquires \& Maintains Attention & $\begin{array}{l}\text { Fall } \\
\text { Spring }\end{array}$ & $\begin{array}{l}1.96(2.30) \\
1.75(2.39)\end{array}$ & 0.55 & $n s$ \\
\hline Asking Questions & $\begin{array}{l}\text { Fall } \\
\text { Spring }\end{array}$ & $\begin{array}{l}2.51(4.38) \\
2.78(3.78)\end{array}$ & 0.61 & $n s$ \\
\hline Behavior Expectations & $\begin{array}{l}\text { Fall } \\
\text { Spring }\end{array}$ & $\begin{array}{l}3.08(3.19) \\
2.10(3.32)\end{array}$ & 1.77 & $n s$ \\
\hline Builds on Responses of Students & $\begin{array}{l}\text { Fall } \\
\text { Spring }\end{array}$ & $\begin{array}{l}1.30(1.85) \\
1.77(1.95)\end{array}$ & 1.78 & $n s$ \\
\hline Challenging Learning Environment & $\begin{array}{l}\text { Fall } \\
\text { Spring }\end{array}$ & $\begin{array}{l}1.25(2.65) \\
1.05(1.83)\end{array}$ & 0.69 & $n s$ \\
\hline Feedback to Many & $\begin{array}{l}\text { Fall } \\
\text { Spring }\end{array}$ & $\begin{array}{l}0.28(0.55) \\
0.44(0.76)\end{array}$ & 1.71 & $n s$ \\
\hline Instructional Activities & $\begin{array}{l}\text { Fall } \\
\text { Spring }\end{array}$ & $\begin{array}{l}1.35(1.94) \\
1.80(2.74)\end{array}$ & 1.37 & $n s$ \\
\hline Many Participate & $\begin{array}{l}\text { Fall } \\
\text { Spring }\end{array}$ & $\begin{array}{l}1.54(2.42) \\
1.39(1.71)\end{array}$ & 0.44 & $n s$ \\
\hline Pacing & $\begin{array}{l}\text { Fall } \\
\text { Spring }\end{array}$ & $\begin{array}{l}0.92(1.33) \\
1.20(1.65)\end{array}$ & 1.32 & $n s$ \\
\hline Peer Evaluation $^{\mathrm{b}}$ & $\begin{array}{l}\text { Fall } \\
\text { Spring }\end{array}$ & $\begin{array}{l}0.00(0.00) \\
0.19(0.68)\end{array}$ & 2.48 & $*$ \\
\hline Positive Feedback & $\begin{array}{l}\text { Fall } \\
\text { Spring }\end{array}$ & $\begin{array}{l}0.67(1.09) \\
0.97(1.30)\end{array}$ & 1.68 & $n s$ \\
\hline Practice Opportunities & $\begin{array}{l}\text { Fall } \\
\text { Spring }\end{array}$ & $\begin{array}{l}1.59(2.29) \\
1.46(1.78)\end{array}$ & 0.43 & $n s$ \\
\hline Rapport & $\begin{array}{l}\text { Fall } \\
\text { Spring }\end{array}$ & $\begin{array}{l}2.01(2.03) \\
1.62(1.86)\end{array}$ & 1.40 & $n s$ \\
\hline
\end{tabular}




\begin{tabular}{|c|c|c|c|c|}
\hline Feedback $^{\mathrm{a}}$ & Semester & $M(S D)$ & $\begin{array}{l}\text { Paired } \\
\text { t-test }\end{array}$ & $p$ \\
\hline Relevant Connections & $\begin{array}{l}\text { Fall } \\
\text { Spring }\end{array}$ & $\begin{array}{l}1.35(1.91) \\
1.84(1.71)\end{array}$ & 1.97 & $*$ \\
\hline Restorative Practices & $\begin{array}{l}\text { Fall } \\
\text { Spring }\end{array}$ & $\begin{array}{l}1.09(1.66) \\
1.13(1.71)\end{array}$ & 0.14 & $n s$ \\
\hline Scans Room & $\begin{array}{l}\text { Fall } \\
\text { Spring }\end{array}$ & $\begin{array}{l}1.73(1.71) \\
1.04(1.33)\end{array}$ & 3.08 & $* *$ \\
\hline SDAIE-UDL & $\begin{array}{l}\text { Fall } \\
\text { Spring }\end{array}$ & $\begin{array}{l}1.51(2.72) \\
1.58(2.27)\end{array}$ & 0.18 & $n s$ \\
\hline Sequencing & $\begin{array}{l}\text { Fall } \\
\text { Spring }\end{array}$ & $\begin{array}{l}1.15(1.43) \\
1.46(2.12)\end{array}$ & 1.36 & $n s$ \\
\hline Strong Voice & $\begin{array}{l}\text { Fall } \\
\text { Spring }\end{array}$ & $\begin{array}{l}1.29(1.37) \\
1.10(1.42)\end{array}$ & 0.91 & $n s$ \\
\hline Student to Student Interactions & $\begin{array}{l}\text { Fall } \\
\text { Spring }\end{array}$ & $\begin{array}{l}1.76(2.56) \\
1.73(2.07)\end{array}$ & 0.07 & $n s$ \\
\hline Student Self-Reflection & $\begin{array}{l}\text { Fall } \\
\text { Spring }\end{array}$ & $\begin{array}{l}0.08(0.31) \\
0.22(0.47)\end{array}$ & 2.36 & $*$ \\
\hline Teachable Moments & $\begin{array}{l}\text { Fall } \\
\text { Spring }\end{array}$ & $\begin{array}{l}0.51(0.85) \\
0.75(1.20)\end{array}$ & 1.52 & $n s$ \\
\hline Technology & $\begin{array}{l}\text { Fall } \\
\text { Spring }\end{array}$ & $\begin{array}{l}0.63(1.00) \\
0.49(1.04)\end{array}$ & 0.88 & $n s$ \\
\hline Timely Feedback & $\begin{array}{l}\text { Fall } \\
\text { Spring }\end{array}$ & $\begin{array}{l}0.57(1.63) \\
0.62(1.02)\end{array}$ & 0.24 & $n s$ \\
\hline Wait Time & $\begin{array}{l}\text { Fall } \\
\text { Spring }\end{array}$ & $\begin{array}{l}0.58(0.96) \\
0.91(1.25)\end{array}$ & 2.02 & $*$ \\
\hline
\end{tabular}

${ }^{\mathrm{a}} n=79$. ${ }^{\mathrm{b}}$ Equal variances not assumed. ${ }^{*} p<.05 . * * p<.01$.

3) What effect does university-supervisors' feedback have on candidates' performance on the summative assessment known as the edTPA?

To determine the effect of feedback on edTPA, we conducted a Univariate General Linear Model and associated t-tests. The sum of the four edTPA Instructional $\mathrm{Ru}-$ brics (see Table 1) served as the dependent variable. Whether or not teacher-candidates received each of 29 types of feedback that university-supervisors provided in the Spring semester (3 months preceding the edTPA) served as independent variables. Peer Evaluation was not included, because none of the teacher-candidates received this type of feedback from university-supervisors in the Spring semester. We conducted a Univariate General Linear Model, because conducting many independent t-tests has a likelihood of producing Type 1 errors. The associated t-tests are reported in Table 5.

Table 5

Difference in Means (SD) on Instructional Portion of the edTPA Based on Feedback

\begin{tabular}{llllll}
\hline Feedback & Given or Not & $n$ & $M(S D)$ & $t$-test & $p$ \\
\hline Academic Language & Given & 37 & $12.67(0.88)$ & 2.27 & $<.05$ \\
& Not Given & 42 & $12.26(0.73)$ & & \\
Acquires \& Maintains Attention & Given & 33 & $12.48(0.71)$ & 0.26 & $n s$ \\
& Not Given & 46 & $12.43(0.91)$ & & \\
\hline
\end{tabular}




\begin{tabular}{|c|c|c|c|c|c|}
\hline Feedback & Given or Not & $\mathrm{n}$ & $\mathrm{M}(\mathrm{SD})$ & t-test & $p$ \\
\hline Behavior Expectations & $\begin{array}{l}\text { Given } \\
\text { Not Given }\end{array}$ & $\begin{array}{l}37 \\
42\end{array}$ & $\begin{array}{l}12.57(0.73) \\
12.36(0.91)\end{array}$ & 1.13 & $n s$ \\
\hline Builds on Responses of Students & $\begin{array}{l}\text { Given } \\
\text { Not Given }\end{array}$ & $\begin{array}{l}45 \\
34\end{array}$ & $\begin{array}{l}12.62(0.78) \\
12.24(0.85)\end{array}$ & 2.10 & $<.05$ \\
\hline Challenging Learning Environment & $\begin{array}{l}\text { Given } \\
\text { Not Given }\end{array}$ & $\begin{array}{l}19 \\
60\end{array}$ & $\begin{array}{l}12.53(0.61) \\
12.43(0.89)\end{array}$ & 0.42 & $n s$ \\
\hline Feedback to Many & $\begin{array}{l}\text { Given } \\
\text { Not Given }\end{array}$ & $\begin{array}{l}10 \\
69\end{array}$ & $\begin{array}{l}12.50(0.77) \\
12.45(0.86)\end{array}$ & 0.18 & $n s$ \\
\hline Instructional Activities & $\begin{array}{l}\text { Given } \\
\text { Not Given }\end{array}$ & $\begin{array}{l}30 \\
49\end{array}$ & $\begin{array}{l}12.60(0.77) \\
12.37(0.86)\end{array}$ & 1.21 & $n s$ \\
\hline Many Participate & $\begin{array}{l}\text { Given } \\
\text { Not Given }\end{array}$ & $\begin{array}{l}32 \\
47\end{array}$ & $\begin{array}{l}12.56(0.72) \\
12.38(0.90)\end{array}$ & 0.94 & $n s$ \\
\hline Modeling & $\begin{array}{l}\text { Given } \\
\text { Not Given }\end{array}$ & $\begin{array}{l}42 \\
37\end{array}$ & $\begin{array}{l}12.52(0.77) \\
12.38(0.89)\end{array}$ & 0.78 & $n s$ \\
\hline Movement Around the Room & $\begin{array}{l}\text { Given } \\
\text { Not Given }\end{array}$ & $\begin{array}{l}28 \\
51\end{array}$ & $\begin{array}{l}12.61(0.69) \\
12.37(0.89)\end{array}$ & 1.21 & $n s$ \\
\hline Pacing & $\begin{array}{l}\text { Given } \\
\text { Not Given }\end{array}$ & $\begin{array}{l}26 \\
53\end{array}$ & $\begin{array}{l}12.38(0.75) \\
12.49(0.87)\end{array}$ & 0.53 & $n s$ \\
\hline Positive Feedback & $\begin{array}{l}\text { Given } \\
\text { Not Given }\end{array}$ & $\begin{array}{l}22 \\
57\end{array}$ & $\begin{array}{l}12.59(0.67) \\
12.40(0.88)\end{array}$ & 0.90 & $n s$ \\
\hline Positive Language & $\begin{array}{l}\text { Given } \\
\text { Not Given }\end{array}$ & $\begin{array}{l}35 \\
44\end{array}$ & $\begin{array}{l}12.54(0.70) \\
12.39(0.92)\end{array}$ & 0.83 & $n s$ \\
\hline Practice Opportunities & $\begin{array}{l}\text { Given } \\
\text { Not Given }\end{array}$ & $\begin{array}{l}31 \\
48\end{array}$ & $\begin{array}{l}12.45(0.81) \\
12.46(0.85)\end{array}$ & 0.35 & $n s$ \\
\hline Rapport & $\begin{array}{l}\text { Given } \\
\text { Not Given }\end{array}$ & $\begin{array}{l}32 \\
47\end{array}$ & $\begin{array}{l}12.56(0.72) \\
12.38(0.90)\end{array}$ & 0.94 & $n s$ \\
\hline Relevant Connections & $\begin{array}{l}\text { Given } \\
\text { Not Given }\end{array}$ & $\begin{array}{l}43 \\
36\end{array}$ & $\begin{array}{l}12.49(0.80) \\
12.42(0.87)\end{array}$ & 0.38 & $n s$ \\
\hline Restorative Practices & $\begin{array}{l}\text { Given } \\
\text { Not Given }\end{array}$ & $\begin{array}{l}29 \\
50\end{array}$ & $\begin{array}{l}12.55(0.78) \\
12.40(0.86)\end{array}$ & 0.78 & $n s$ \\
\hline Scans Room & $\begin{array}{l}\text { Given } \\
\text { Not Given }\end{array}$ & $\begin{array}{l}26 \\
53\end{array}$ & $\begin{array}{l}12.50(0.65) \\
12.43(0.91)\end{array}$ & 0.33 & $n s$ \\
\hline Sequencing & $\begin{array}{l}\text { Given } \\
\text { Not Given }\end{array}$ & $\begin{array}{l}31 \\
48\end{array}$ & $\begin{array}{l}12.45(0.57) \\
12.46(0.97)\end{array}$ & 0.04 & $n s$ \\
\hline Strong Voice & $\begin{array}{l}\text { Given } \\
\text { Not Given }\end{array}$ & $\begin{array}{l}30 \\
49\end{array}$ & $\begin{array}{l}12.53(0.73) \\
12.41(0.89)\end{array}$ & 0.65 & $n s$ \\
\hline Student to Student Interactions & $\begin{array}{l}\text { Given } \\
\text { Not Given }\end{array}$ & $\begin{array}{l}42 \\
37\end{array}$ & $\begin{array}{l}12.50(0.80) \\
12.41(0.86)\end{array}$ & 0.50 & $n s$ \\
\hline Student Self-Reflection & $\begin{array}{l}\text { Given } \\
\text { Not Given }\end{array}$ & $\begin{array}{l}4 \\
75\end{array}$ & $\begin{array}{l}13.00(1.41) \\
12.43(0.79)\end{array}$ & 1.36 & $n s$ \\
\hline
\end{tabular}


Table 5 Cont.

\begin{tabular}{llllll}
\hline Feedback & Given or Not & $\mathrm{n}$ & $\mathrm{M}(\mathrm{SD})$ & $\mathrm{t}$-test & $p$ \\
\hline Technology & Given & 13 & $12.69(0.75)$ & 1.13 & $n s$ \\
& Not Given & 66 & $12.41(0.84)$ & & \\
Timely Feedback & Given & 15 & $12.53(0.74)$ & 0.40 & $n s$ \\
& Not Given & 64 & $12.44(0.85)$ & & \\
Wait Time & Given & 23 & $12.61(0.78)$ & 1.05 & $n s$ \\
& Not Given & 56 & $12.39(0.85)$ &
\end{tabular}

Findings show that teacher-candidates who received feedback on skills "academic language" and "builds on responses of students" performed statistically significantly better on the Instruction Rubrics of the edTPA than candidates who did not receive feedback on those skills. These findings highlight the associative relationship supervisors' feedback play in the development of candidates' teaching skills.

\section{Discussion, Future Research, and Implications}

Empirical research suggests that video-based coaching tools have the potential to develop proper teaching behaviors in novice teachers (Kennedy \& Lees, 2016) and improve K-12 students' academic and behavioral outcomes (Gregory et al., 2017). These studies, however, do not report the mechanism or process by which video-based coaching supports teacher-candidates? With this question in mind, our research examined feedback provided by university-supervisors via a video-based coaching tool to elementary teacher-candidates over the course of an academic year. We were particularly interested in what pedagogical skills supervisors were using to help teacher-candidates notice aspects of their teaching. The video-based coaching tool, Sibme, allowed supervisors to identify specific pedagogical skills when providing feedback to teacher-candidates. These pedagogical skills were aligned to state standards and to a national performance assessment that is widely used throughout the United States, the edTPA.

Two theoretical constructs guided our thinking about feedback. First, feedback needs to be positive and specific (Hattie \& Timperley, 2007; Shute, 2008; Voerman et al., 2012). Our findings suggest that university-supervisors were liberal with dispensing "bright spots" for both fall and spring semesters. They, however, provided more "ideas" in the fall to teacher candidates than the spring semester. Additionally, university-supervisors asked fewer questions that promoted critical thinking among teachercandidates across both semesters. These findings align with previous research that describe university-supervisors as tending to provide positive forms of feedback, as opposed to constructive support (Kolman, 2018; Ritter et al., 2011; Schwartz, Walkowiak, Poling, Richardson, \& Polly, 2018). Being positive may be a tool supervisors use to bolster teacher-candidates' confidence and motivation [20], while perhaps avoiding negative reactions from candidates. The skills needed to become an effective teacher, however, are complex and require much needed support from teachers in the field as well as university-supervisors. Therefore, constructive feedback provides teacher-candidates opportunities to correct and practice necessary skills to be effective in classrooms. Moreover, constructive feedback enables teacher-candidates to be persist in supporting their students when teaching or classroom experiences become challenging. University-supervisors, as a result, must establish good rapport that builds trust with teacher candidates, so that they can feel safe to make mistakes and be supported to develop as teaching professionals (Cummins, 2004; Nolan, 2013).

Our data also reports that university-supervisors offered feedback that focused on specific pedagogical skills. According to Goodwin's (1994) professional vision and Mason's (2002) noticings, the level of specificity plays a role in the effectiveness of feedback. The findings from our study suggest that most university-supervisors offered specific feedback that addressed teacher-candidates' classroom management and instructional activities. Universitysupervisors frequently shared with teacher-candidates the importance of setting appropriate behavioral expectations and using positive language when talking to students. Likewise, university-supervisors provided feedback on modeling and asking questions with a focus on instructional activities. These skills are important for novice teachers to develop because they focus on what Kolman (2018) refers to as links to the lesson plan. Stating clear expectations, modeling, and asking questions are teacher skills associated with lesson planning and design. In short, these types of feedback focus mainly on the activity of teaching (i.e., what teachers do) rather than the outcomes of teaching (what students learn). Kolman (2018) further states that these types of skills are especially offered as feedback to teacher-candidates whom supervisors perceive as struggling. When a university-supervisor supports a teacher candidate whom they perceive to be advanced, feedback tend to focus on student learning. In short, university-supervisors use their professional vision to notice what they deem important pedagogical skills, especially for teacher-candidates whom they perceive need little remediation.

In addition to examining on what skills university-supervisors focused their feedback, we also investigated whether university-supervisors modulated their feedback to support differential needs of their teacher-candidates. At the beginning of student-teaching experiences, teachercandidates may require different types of feedback than later in the program, as they develop more teaching skills. Our findings highlight that for the most part, universitysupervisors maintained the same level of feedback on most skills throughout the academic year. For example, university-supervisors provided mostly positive feedback for the 
entirety of the clinical field experience in the schools. The level of constructive feedback (i.e., asking questions) also did not fluctuate overtime. University-supervisors did offer more ideas and recommendations to candidates toward the beginning of the year than the latter part of the teachercredential program. This reflects supervisors' promoting candidates' gradual acquisition of responsibility (Pearson \& Gallagher, 1983; Webb et al., 2019). When teacher candidates are new in the classroom, supervisors highlight certain skills. As candidates become more experienced, these skills become less relevant to supporting candidates' development.

With regard to pedagogical skills, university-supervisors modulated the amount of feedback associated with noticing classroom management and student-centered skills during the academic year. Toward the start of the academic year, university-supervisors provided more feedback that focused on skills such as moving around the room or scanning the room than toward the end of the program. Again, this reflects candidates' gradual acquisition of responsibility with regards to classroom management skills. Supervisors' feedback focused more on classroom management in the fall semester and less so in the spring semester.

As candidates gained more independence with classroom management, supervisors were able to provide feedback that focused more on student-centered skills. Teacher-candidates were encouraged to notice specific pedagogical skills such as promoting relevant connections between the lesson and their students, fostering students to reflect on their own skills and to evaluate their peers, and considering the importance of wait time when asking questions. This shift supports candidates in becoming more constructivist, with greater attention to student-centered skills (Schwartz et al., 2018). Supervisors' feedback may reflect the developmental progression of teacher-candidates, but it also may reflect supervisors' perceptions of their candidates. Since university-supervisors focused on student-centered pedagogies with some teacher candidates rather than all, perhaps university-supervisors perceived select candidates as advanced enough to receive feedback about student-centered pedagogies (Ritter et al., 2011). Another possible explanation may be that supervisors are modulating their focus because of the edTPA, which candidates complete near the conclusion of the program. Thus, as student-centered activities increased in preparation for the edTPA, so did feedback on student-centered skills. Okraski and Kissau (2018) suggests that university-structured activities provide support for candidates in developing their understanding of the focus and purpose of the edTPA. University-supervisors use their professional vision to determine what skills should be noticed, when should the skills be noticed, and who is eligible to notice them. Therefore, university-supervisors play a significant role in what is noticed by teacher-candidates. Does this role lead to tangible outcomes for the candidate?

Thus, we examined the effect of university-supervisors' feedback on teacher-candidates' edTPA performance. If university-supervisors modulate their feedback in order to support candidates on the edTPA, does their feedback influence candidates' edTPA performance? Results show that when supervisors offered feedback on student-centered pedagogical skills, candidates scored higher on the edTPA. Research findings that identify connections between university-supervisors' support and performance on high-stakes assessments like the edTPA are rare. Yet, in our research study university-supervisors helped candidates notice some relevant skills that, according to our data, showed an association with increased performance on the edTPA. It is likely that feedback on classroom management skills do not relate to edTPA scores mainly because edTPA focuses more on student-centered skills rather than class management. Of those student-centered skills, academic language use and building on students' responses were significantly connected with edTPA scores. That is, when university-supervisors encouraged candidates to notice these stated skills, their performance on the edTPA was significantly higher than those candidates who were not encouraged to notice these skills.

These findings suggest that university-supervisors' feedback can have a measurable effect on candidates' pedagogical development. Moreover, our findings highlight that supervisors do not just provide emotional support to candidates (Donovan \& Cannon, 2018), but rather, university-supervisors can have a profound role in helping candidates notice the professional vision associated with effective teaching. Performance-based assessments, like the edTPA, can help identify that professional vision. Videobased coaching enables supervisors to support teacher-candidates' noticings of pedagogical skills associated with the teaching performance and effective teaching (Choppin \& Meuwissen, 2017).

\section{Future Research}

This study reports findings about the feedback university-supervisors provide to teacher-candidates and how that feedback supports candidates' performance on a national performance-based assessment. Our findings show a connection between feedback to teacher-candidates and their edTPA performance. Our findings, however, do not confirm that the feedback university-supervisors provided teacher-candidates enabled them to perform better on the edTPA. To confirm such a cause and effect relationship, future research should not only examine the tags used to help candidates notice specific pedagogical skills, but also the written feedback as well as face to face conversations explaining the meaning behind the tags.

Another focus of future research should be on ensuring reliability among labeled skills associated with university-supervisors' feedback. While some supervisors addressed student-centered skills, the interpretation of those skills by individual supervisors could be different. Future research should examine how supervisors are trained to provide feedback and the calibration of feedback labels among supervisors. Understanding how supervisors' use video-based coaching tools to support and develop future teachers is an important area of research to conduct. Videobased coaching offers many newly available opportunities. The tool, however, is only as good as the users' attitudes toward it (Authors, 2018). Defining the context for this video-based coaching tool to be useful and best practices for how to effectively use this tool should be part of future research.

\section{Implications}

Video-based coaching can change the role of university-supervisors from support providers who provide 
future teachers with emotional support to coaches who use their expertise to help future teachers notice essential pedagogical skills within their instructional practice. Moreover, this new approach to coaching teacher-candidates can result in the development and implementation of best prac-

\section{REFERENCES}

1. Koerner, M., Rust, F., \& Baumgartner, F. (2002). Exploring roles in student teaching placements. Teacher Education Quarterly, 29 (2), 35-58 [in English].

2. Authors. (2017). [Title omitted for blind review]. Teacher Education Quarterly [in English].

3. Authors. (2018). [Title omitted for blind review]. J Technology and Teacher Education [in English].

4. Tan, A. L., \& Towndrow, P. A. (2009). Catalyzing student-teacher interactions and teacher learning in science practical formative assessment with digital video technology. Teaching and Teacher Education, 25(1), 6167 [in English].

5. Hamilton, E. R. (2012). Video as a metaphorical eye: Images of positionality, pedagogy, and practice. College Teaching, 60(1), 10-16 [in English].

6. van Es, E. A., \& Sherin, M. G. (2008). Mathematics teachers" "learning to notice" in the context of a video club. Teaching and Teacher Education, 24(2), 244-276 [in English].

7. Marsh, B., \& Mitchell, N. (2014). The role of video in teacher professional development. Teacher Development, 18(3), 403-417 [in English].

8. Sun, J., \& Van Es, E. A. (2015). An Exploratory Study of the Influence That Analyzing Teaching Has on Preservice Teachers' Classroom Practice. J of Teacher Education, 66(3), 201-214 [in English].

9. Voerman, L., Meijer, P. C., Korthagen, F. A., Simon, R. J. (2012). Types and frequencies of feedback interventions in classroom interaction in secondary education. Teaching and Teacher Education, 28(8) [in English].

10. Hattie, J., \& Timperley, H. (2007). The power of feedback. Rev of Educ. Research, 77(1), 81-112 [in English].

11. Shute, V. J. (2008). Focus on formative feedback. Rev of Educ. Research, 78(1), 153-189 [in English].

12. Goodwin, C. (1994). Professional vision. American Anthropologist, 96(3), 606-633 [in English].

13. Mason, J. (2002). Researching Your Own Practice: The Discipline of Noticing. Psychology Press [in English].

14. Lefstein \& Snell. (2011). Professional vision and the politics of teacher learning. Teaching and Teacher Education, 27(3), 505-514 [in English].

15. Stürmer, K., Könings, K., \& Seidel, T. (2013). Declarative knowledge and professional vision in teacher education: Effect of courses in teaching and learning. British J of Educational Psychology,83(3), 467-483 [in English].

16. Wu, C. C., \& Lee, G. C. (2004). Use of computermediated communication in a teaching practicum course. tices that can be measured through performance-based assessment. Video-based coaching by university-supervisors has the potential to improve teacher education and, by extension, student achievement.

International Journal of Science and Mathematics Education, 2(4), 511-528 [in English].

17. Kennedy, A. S., \& Lees, A. T. (2016). Preparing undergraduate pre-service teachers through direct and video-based performance feedback and tiered supports in early Head Start. Early Childhood Education Journal, 44(4), 369-379 [in English].

18. Gregory, A., Ruzek, E., Hafen, C. A., Mikami, A. Y., Allen, J. P., \& Pianta, R. C. (2017). My teaching partner-secondary: A video-based coaching model. Theory Into Practice, 56(1), 38-45 [in English].

19. Kolman, J. S. (2018). Clinical supervision in teacher preparation exploring the practices of universityaffiliated supervisors. Action in Teacher Education, 40(3) [in English].

20. Ritter, J. K., Powell, D., Hawley, T. S., \& Blasik, J. (2011). Reifying the ontology of individualism at the expense of democracy: An examination of university supervisors' written feedback to student teachers. Teacher Education Quarterly, 38(1) [in English].

21. Schwartz, C., Walkowiak, T. A, Polin, L., Richardson, K., \& Polly, D. (2018). The nature of feedback given to elementary student teachers from university supervisors after observations of mathematics lessons. Mathematics Teacher Education and Development, 20(1), 6285 [in English].

22. Cummins, L. (2004). The pot of gold at the end of the rainbow: Mentoring in early childhood education. Childhood Education, 80(5), 254 [in English].

23. Nolan, A., Morrissey, A. M., \& Dumenden, I. (2013). Expectations of mentoring in a time of change: Views of new and professionally isolated early childhood teachers in Victoria, Australia. Early Years, 33(2), 161171 [in English].

25. Pearson, P. D., \& Gallagher, M. C. (1983). The instruction of reading comprehension. Contemporary Educational Psychology, 8(3), 317-344 [in English].

26. Webb, S., Massey, D., Goggans, M., \& Flajole, K. (2019). Thirty-five years of the gradual release of responsibility: Scaffolding toward complex and responsive teaching. Reading Teacher,73(1), 75-83 [in English].

27. Okraski, C. V., \& Kissau, S. P. (2018). Impact of content-specific seminars on candidate edTPA preparation and performance. Foreign Language Annals, 51(3), 685705 [in English].

28. Donovan, M. K., \& Cannon, S. O. (2018). The university supervisor, edTPA, and the new making of the teacher. Education Policy Analysis Archives 26(28), 1-26 [in English].

29. Choppin, J., \& Meuwissen, K. (2017). Threats to validity in the edTPA video component. Action in Teacher Education, 39(1), 39-53 [in English]. 
Естелла Чижик, професор педагогічної освіти, Олександр Чижик, професор педагогічної освіти,

Брайан Берджсес, студент кафедри психології,

Мелін Танмаджс, бакалавр з психології,

Деррік Сенг, студент кафедри психології,

Мірна Ернандес, бакалавр з психології, Державний університет Сан-Дієго, вул. Кемпаніл Драйв, 5500, Сан-Дієго, Каліфорнія, США

\section{ВІДЕО-ТРЕНІНГ ДЛЯ ЗАБЕЗПЕЧЕННЯ ПРОФЕСІЙНОГО РОЗВИТКУ ВЧИТЕЛІВ ПОЧАТКОВИХ КЛАСІВ}

Програми педагогічної освіти прагнуть удосконалити свою клінічну практику для майбутніх вчителів. Вiдео-тренінг дозволяє університетським фахівцям можливість надавати конструктивний зворотний зв'язок майбутнім вчителям. Більшість досліджень відео-тренінгу зосереджуються на тому, як програма допомагає майбутнім вчителям помітити аспекти їхнього навчання. Мало досліджень присвячено типам університетських зворотних зв'язків, які забезпечують своїх майбутніх вчителів. Ця робота досліджує як здійснюється університетський зворотний зв'язок і яким чином він змінює курс програми та його вплив на підсумкову оцінку на основі ефективності. На основі зворотного зв'язку 16 університетських фахівців для 124 майбутніх вчителів початкових класів, встановлено, що університетські зворотні зв'язки мають тенденцію бути більш позитивістськими, ніж конструктивними. Навички вибору, на яких фахівці зосереджувались, модулювались у часі та асоціюються з роботою майбутніх вчителів на підсумковій оцінці ефективності. Висновки цього дослідження свідчать про те, що університетські фахівці можуть за допомогою відео-тренінгу суттєво впливати на навчальну продуктивність майбутніх вчителів.

Ключові слова: педагогічна освіта, майбутні вчителі, університетські фахівці, навчання на основі відео, зворотний зв'язок, оцінка на основі ефективності.

Подано до редакиіï 20.09.2019

УДК $378.147+37.026$

DOI: https://doi.org/10.24195/2414-4665-2019-4-2

Раиса Мартынова, доктор педагогических наук, профессор, Государственное учреждение «Южснукраинский национальный педагогический университет имени К. Д. Уиинского», ул. Старопортофрансковская, 26, г. Одесса, Украина

\section{ВИДЫ ДИДАКТИЧЕСКИХ МОДЕЛЕЙ ПРОЦЕССА ОБУЧЕНИЯ}

В статье обосновывается возможность совершенствования результативности процесса обучению любой дисциплины путем его предварительного моделирования. Установлено, что любая дидактическая модель состоит из трех блоков: организачионного, который представляет собой этапь обучения; содержательного, включающего звенья: иели обучения, элементы предмета обучения и компоненты содержания обучения; процессуального, включающего звенья: методы обучения, средства обучения, контроль результатов обучения. Такая модель обладает следующими свойствами системности: иелостностью, иерархичностью, эмерджетностью, функциональностью, синергетичностью. Ее валидность определяется концептуально, то есть учетом: диалектических законов познания окружающей действительности; синергетических законов взаимодействия больших и мальх величин, которые в педагогике представляют собой огромные массивы информации во многих областях знаний и способы ее компрессии для возможного по времени ее познания; психологических закономерностей усвоения запрограммированного материала и дидактических принципов организации процесса обучения. Ее валидность также определяется критериально - путем использования следуюших критериев: сравнительно-целеполагающего, сравнительно-тематического, структурно-сравнительного, сравнительно- 